\title{
RECURSIONS FOR CERTAIN BIVARIATE COUNTING DISTRIBUTIONS AND THEIR COMPOUND DISTRIBUTIONS
}

\author{
By Ole Hesselager \\ Laboratory of Actuarial Mathematics \\ University of Copenhagen
}

\begin{abstract}
We consider three classes of bivariate counting distributions and the corresponding compound distributions. For each class we derive a recursive algorithm for calculating the bivariate compound distribution.
\end{abstract}

\section{KEYWORDS}

Bivariate counting distributions; Compound distributions; Recursions; Bivariate mixed Poisson.

\section{INTRODUCTION}

In this paper we develop recursive algorithms for bivariate compound distributions of the type

$$
g(x, y)=\sum_{n, m=0}^{\infty} p(n, m) f_{1}^{*_{n}}(x) f_{2}^{*_{m}}(y), x, y=0,1, \ldots,
$$

which is the joint distribution of

$$
(X, Y)=\left(\sum_{i=0}^{N} U_{i}, \sum_{i=0}^{M} V_{i}\right)
$$

where $(N, M)$ has a probability function (pf)

$$
p(n, m)=\mathrm{P}(N=n, M=m),
$$

and all the severities $U_{i}, V_{i}$ are mutually independent and independent of $(N, M)$ with pf's

$$
f_{1}(u)=\mathrm{P}\left(U_{i}=u\right), f_{2}(v)=\mathrm{P}\left(V_{i}=v\right)
$$

on the non-negative integers.

ASTIN BULLETIN, Vol. 26, No. I, 1996, pp. 35-52 
For a counting variable $K$ we write $K \sim R_{1}(a, b)$ when its pf $q$ satisfies the recursion

$$
q(k)=\left(a+\frac{b}{k}\right) q(k-1), \quad k \geq 1,
$$

and $K \sim R_{1}$ means that (1.3) holds for some constants $a$ and $b$.

For a compound variable $X$ with counting distribution $R_{1}(a, b)$ and severity distribution $f$ we remind of the fact that the pf of $X$ can be calculated from PANJER's (1981) recursive formula

$$
g(x)=\frac{1}{1-a f(0)} \sum_{u=1}^{x}\left(a+\frac{b u}{x}\right) f(u) g(x-u),
$$

when $f$ is concentrated on the non-negative integers. Also the identity

$$
\frac{1}{n+i} f^{*(n+i)}(x)=\sum_{u=1}^{x} \frac{u}{i x} f^{*_{i}}(u) f^{*_{n}}(x-n),
$$

from SCHRÖTER (1990, Lemma 1) will be used in the following.

In sections 2, 3 and 4 we consider three different models:

- Model A. With $K=N+M$ it holds that $(N \mid K=k) \sim \operatorname{Binomial}\left(k, \rho_{1}\right)$, and $K \sim R_{1}(a, b)$.

- Model B. $N=R_{0}+R_{1}$ and $M=R_{0}+R_{2}$, where $R_{0}, R_{1}$ and $R_{2}$ are mutually independent and $R_{j} \sim R_{1}\left(a_{j}, b_{j}\right)$.

- Model C. $N$ and $M$ are conditionally independent given $\theta=\vartheta$ and Poisson distributed with parameters $\vartheta \lambda_{1}$ and $\vartheta \lambda_{2}$, respectively. The parameter $\theta$ has a density $u$ which satisfies

$$
\frac{d}{d \vartheta} \log u(\vartheta)=\frac{\sum_{i=0}^{k} b_{i} \vartheta^{i}}{\sum_{i=0}^{k} a_{i} \vartheta^{i}} .
$$

We also consider the marginal pf's

$$
g(x)=\sum_{y=0}^{\infty} g(x, y), \quad p(n)=\sum_{m=0}^{\infty} p(n, m),
$$

for $X$ and $N$, and the conditional pf's

$$
g(y \mid x)=g(x, y) / g(x), \quad p(m \mid n)=p(n, m) / p(n),
$$

for $(Y \mid X=x)$ and $(M \mid N=n)$. In particular, we derive recursions for the conditional moments,

$$
\mu_{l, x}=\mathrm{E}\left(Y^{l} \mid X=x\right)=\sum_{y=0}^{\infty} y^{l} g(y \mid x),
$$

based on the auxiliary functions

$$
\tilde{\mu}_{l, x}=g(x) \mu_{l, x} .
$$


The bivariate recursions are of interest in prediction problems involving the conditional pf $g(y \mid x)$ of $Y$, given that $X=x$ has been observed. Using the recursions derived in this paper this will involve the calculation of $g(u, y)$ for all $u=0, \ldots, x$ and $y=0,1, \ldots$ It should be noted that if one is only interested in the distribution of $X+Y$, then there are simpler ways of calculating this distribution than via $g(x, y)$, and the bivariate recursions should not be used in this case.

Model A has a natural application in claims reserving where $K$ denotes the total number of claims incurred in a fixed exposure period. If $W_{i}, i=1, \ldots, K$, denote the waiting times until notification, which can be assumed to be iid and independent of $K \sim R_{1}(a, b)$, then the numbers of reported and outstanding claims at time $\tau$,

$$
N=\sum_{i=1}^{K} I\left(W_{i} \leq \tau\right), \quad M=\sum_{i=1}^{K} I\left(W_{i}>\tau\right)
$$

satisfy assumption A with $\rho_{1}=\mathrm{P}\left(W_{i} \leq \tau\right)$.

The recursion obtained in Section 2 can be viewed as a bivariate version of the Panjer recursion).

The situation with a binomial distribution of $N$ given $N+M$ as assumed in model A arises in a variety of insurance problems. Consider for instance the case where a stop-loss contract with retention limit $d$ has been written for a one-year period $[0,1]$. At time $t \in[0,1]$ the aggregate claim amount has reached the level $X=x$, and the problem is to determine the probability $\sum_{y>d-x} g(y \mid x)$ that the final claim amount $X+Y$ will exceed the limit $d$, given the information $X=x$ (or to determine the expected reinsurance recoveries $\sum_{y>d-x}(x+y-d) g(y \mid x)$ in this case). If the claim occurrences are generated by a mixed Poisson process with (random) intensity $\theta \eta(s), 0 \leq s \leq 1$, then the claim numbers $(N, M)$ occurring in $[0, t]$ and $(t, 1]$ satisfy the binomial assumption of model A with $\rho_{1}=\int_{0}^{t} \eta(s) d s /$ $\int_{0}^{1} \eta(s) d s$. The recursion derived in Section 2 is then applicable if $\theta$ has a gamma distribution, in which case the total number of claims $K=N+M$ has a negative binomial distribution $\left(\in R_{1}\right)$, and of course in the Poisson case where $\theta$ is degenerate. More generally, if the mixing distribution satisfies the condition given in model C, we may use the recursion derived in Section 4 with $\lambda_{1}=\int_{0}^{t} \eta(s) d s$ and $\lambda_{2}=\int_{t}^{1} \eta(s) d s$. Another application of model $\mathrm{C}$ arises in connection with customer based rating where a customer with unknown risk characteristics represented by $\theta$ has reported a total claim amount $X=x$ on the existing policies. For a new policy, this customer will report a claim amount of $Y$ during the next year, and this policy can then be rated on the basis of the experience $X=x$ using the conditional pf $g(y \mid x)$.

The class of mixed Poisson distributions considered in Section 4 were investigated by Willmot (1993) and Hesselager (1993) in the univariate case, and the recursions derived in Section 4 give a bivariate extension of their results.

Model B uses a standard way of constructing bivariate distributions (see e.g. KOCHERLAKOTA et al. 1992), which is useful in risk theory when two risk classes are affected by the same events: Let $R_{1}$ and $R_{2}$ denote the numbers of events causing a claim in class 1 and class 2 , respectively, and let $R_{0}$ denote the number of events causing a claim to both classes. 
Model B was studied in the Poisson case where $a_{j}=0$ for $j=0,1,2$ by TEICHER (1954), who obtained a recursion for the bivariate pf $p(n, m)$. In Section 3 we extend the result of TEICHER (1954) to the more general case where $R_{j} \sim R_{1}$, and derive a recursion for the corresponding compound pf $g(x, y)$.

2. A BIVARIATE VERSION OF THE PANJER RECURSION

With $K=N+M$ we consider the following model:

A. The conditional distribution of $N$ given $K$ is binomial,

$$
\mathrm{P}(N=n \mid K=k)=\left(\begin{array}{l}
k \\
n
\end{array}\right) \rho_{1}^{n} \rho_{2}^{k-n}, \rho_{1}+\rho_{2}=1,
$$

and $K \sim R_{1}(a, b)$.

\subsection{Bivariate distributions}

Let

$$
\varphi(s, t)=\mathrm{E}\left[s^{N} t^{M}\right]=\sum_{n, m \geq 0} p(n, m) s^{n} t^{m}
$$

denote the pgf for $(N, M)$, and let $\psi(s)=E s^{K}$ be the pgf for $K=N+M$. From the assumption (2.1) we find that

$$
\begin{aligned}
\varphi(s, t) & =\mathrm{EE}\left[s^{N} t^{M} \mid K\right]=\mathrm{E}\left[t^{K} \mathrm{E}\left[(s / t)^{N} \mid K\right]\right] \\
& =\mathrm{E}\left[t^{K}\left(\rho_{1}(s / t)+\rho_{2}\right)^{K}\right]=\psi\left(\rho_{1} s+\rho_{2} t\right),
\end{aligned}
$$

where we have made use of the fact that the pgf for the binomial distribution with parameters $(k, \rho)$ is $(\rho z+(1-\rho))^{k}$. When $K \sim R_{1}(a, b)$ we also remind of the fact that the $\operatorname{pgf} \psi$ satisfies the differential equation

$$
(1-a s) \psi^{\prime}(s)=(a+b) \psi(s) \text {. }
$$

From this we readily obtain a recursion for the bivariate pf $p(n, m)$ :

Theorem 2.1: Under condition $A$ it holds that

$$
\begin{aligned}
& p(n, m)=\rho_{1}\left(a+\frac{b}{n}\right) p(n-1, m)+a \rho_{2} p(n, m-1), n \geq 1 \\
& p(n, m)=\rho_{2}\left(a+\frac{b}{m}\right) p(n, m-1)+a \rho_{1} p(n-1, m), m \geq 1
\end{aligned}
$$

with $p(n,-1)=p(-1, m)=0$.

Proof. Differentiating (2.2) with respect to $s$ yields

$$
\left(1-a \rho_{1} s-a \rho_{2} t\right) \frac{d}{d s} \varphi(s, t)=(a+b) \rho_{1} \varphi(s, t),
$$


and with $\varphi(s, t)=\sum_{n, m \geqslant 0} p(n, m) s^{n} t^{m}$ we compare the coefficients of $s^{n-1} t^{m}$ in this equation for $n \geq 1$ to obtain

$$
n p(n, m)-a \rho_{1}(n-1) p(n-1, m)-a \rho_{2} n p(n, m-1)=(a+b) \rho_{1} p(n-1, m) \text {. }
$$

This proves the first relation, and the second follows by symmetry.

QED

Theorem 2.1 gives the following recursion for $g(x, y)$ :

Theorem 2.2: Under condition A it holds that

$$
g(0,0)=\psi\left(\rho_{1} f_{1}(0)+\rho_{2} f_{2}(0)\right),
$$

where $\psi(z)$ is the pgf for $K$. For $x \geq 1$ it holds that

(2.5) $g(x, y)=\rho_{1} \sum_{u=0}^{x}\left(a+\frac{b u}{x}\right) f_{1}(u) g(x-u, y)+a \rho_{2} \sum_{v=0}^{y} f_{2}(v) g(x, y-v)$,

and for $y \geq 1,1$

(2.6) $g(x, y)=\rho_{2} \sum_{v=0}^{y}\left(a+\frac{b v}{y}\right) f_{2}(v) g(x, y-v)+a \rho_{1} \sum_{u=0}^{x} f_{1}(u) g(x-u, y)$.

Proof. The initial value is

$$
g(0,0)=\sum_{n, m=0}^{\infty} p(n, m) f_{1}(0)^{n} f_{2}(0)^{m}=\varphi\left(f_{1}(0), f_{2}(0)\right)
$$

and (2.4) follows from (2.2). Using (1.5) for $i=1$ yields

$$
\begin{aligned}
& \sum_{n=1}^{\infty} \sum_{m=0}^{\infty} \frac{1}{n} p(n-1, m) f_{1}^{* n}(x) f_{2}^{* m}(y) \\
& \quad=\sum_{n, m=0}^{\infty} \frac{1}{n+1} p(n, m) f_{1}^{*(n+1)}(x) f_{2}^{* m}(y)=\sum_{u=1}^{x} \frac{u}{x} f_{1}(u) g(x-u, y) .
\end{aligned}
$$

For $x \geq 1$ we then obtain from Theorem 2.1 that

$$
\begin{aligned}
g(x, y) & =\sum_{n=1}^{\infty} \sum_{m=0}^{\infty} p(n, m) f_{1}^{* n}(x) f_{2}^{* m}(y) \\
& =\sum_{n=1}^{x} \sum_{m=0}^{x}\left[\rho_{1}\left(a+\frac{b}{n}\right) p(n-1, m)+a \rho_{2} p(n, m-1)\right] f_{1}^{* n}(x) f_{2}^{* m}(y) \\
& =\rho_{1} \sum_{u=0}^{x}\left(a+\frac{b u}{x}\right) f_{1}(u) g(x-u, y)+\rho_{2} a \sum_{v=0}^{y} f_{2}(v) g(x, y-v) .
\end{aligned}
$$

The second identity (2.6) follows by symmetry.

The bivariate compound distribution is calculated recursively from (2.5) or (2.6) by collecting the terms involving $g(x, y)$ on the left-hand side. It is seen that the 
number of multiplications involved with the calculation of $g(u, v)$ for $(u, v) \leq(x, y)$ is of order $O(x y(x+y))$. If the severity distributions $f_{1}$ and $f_{2}$ have bounded supports, as will typically be the case in practical applications, the number of multiplications will be of order $O(x y)$.

By summing (2.5) over $y \geq 0$ we obtain the Panjer recursion (1.4) for the marginal distribution of $X$, and the recursion obtained from Theorem 2.2 may therefore be viewed as a bivariate extension of the Panjer recursion.

\subsection{Marginal and conditional distributions}

Theorem 2.1 can also be used to identify the marginal and conditional distributions of $(N, M)$. We have the following:

Theorem 2.3 Under condition A it holds that

$$
\begin{gathered}
N \sim R_{1}\left(a \frac{\rho_{1}}{1-a \rho_{2}}, b \frac{\rho_{1}}{1-a \rho_{2}}\right), \\
(M \mid N=n) \sim R_{1}\left(a \rho_{2},(b+a n) \rho_{2}\right) .
\end{gathered}
$$

Proof. By summing the first relation in Theorem 2.1 over $m \geq 0$ we find that

$$
p(n)=\frac{\rho_{1}}{1-a \rho_{2}}\left(a+\frac{b}{n}\right) p(n-1), n \geq 1,
$$

which proves the first assertion. For the conditional pf $p(m \mid n)$ we observe from the second relation in Theorem 2.1 that

$$
\frac{p(m \mid n)}{p(m-1 \mid n)}=\frac{p(n, m)}{p(n, m-1)}=\rho_{2}\left(a+\frac{b}{m}\right)+a \rho_{1} \frac{p(n-1, m)}{p(n, m-1)}, m \geq 1 .
$$

Since $p(n, m)=q(n+m)\left(\begin{array}{c}n+m \\ n\end{array}\right) \rho_{1}^{n} \rho_{2}^{m}$, where $q$ is the pf for $K$, we have that

$$
\frac{p(n-1, m)}{p(n, m-1)}=\frac{n}{m} \frac{\rho_{2}}{\rho_{1}}
$$

which proves the result.

The class $R_{1}$ contains the binomial distributions $(a<0)$, the Poisson distributions $(a=0)$, and the negative binomial distributions $(0<a<1)$. It is seen from Theorem 2.3 that the marginal and conditional distributions are binomial, Poisson or negative binomial when the distribution of $K$ is binomial, Poisson or negative binomial, respectively. In particular, it is seen that $N$ and $M$ are independent in the Poisson case $(a=0)$.

Note also from Theorem 2.3 that the marginal distribution of $X$ and the conditional distribution of $(Y \mid N=n)$ can be calculated by use of the Panjer recursion (1.4). 
In order to calculate the conditional pf $g(u \mid x)$ for $y=0, \ldots, y_{\max }$, one needs to calculate $\operatorname{pf} g(u, v)$ for all $(u, v) \leq\left(x, y_{\max }\right)$. In some cases one may settle for an approximation to this distribution, based on the conditional moments $\mu_{k, x}$.

Theorem 2.4 With $e_{j}=\sum_{\nu=0}^{x} v^{j} f_{2}(v)$, it holds that

$$
\left(1-a \rho_{2}\right) \tilde{\mu}_{l, x}=a \rho_{1} \sum_{u=0}^{x} f_{1}(u) \tilde{\mu}_{l, x-u}+a \rho_{2} \sum_{i=0}^{l-1} c(l, i) \tilde{\mu}_{i, x} e_{l-i},
$$

where $c(l, i)=a\left(\begin{array}{l}l \\ i\end{array}\right)+b\left(\begin{array}{c}l-1 \\ i\end{array}\right)$.

Theorem 2.4 follows from Theorem 2.2 by straightforward calculations, and the details can be found in Appendix A. With $\tilde{\mu}_{l, x}$ given by Theorem 2.4, and the marginal pf $g(x)$ of $X$ being calculated from the Panjer recursion, we then have a recursive procedure for obtaining the conditional moments $\mu_{l, x}=\tilde{\mu}_{l, x} / g(x)$.

Note that $\mathrm{E} Y^{l}=\sum_{x} \tilde{\mu}_{l, x}$. By summing (2.7) over $x$ we obtain that

$$
\mathrm{E} Y^{l}=\frac{a \rho_{2}}{1-a} \sum_{i=0}^{l-1} c(l, i) E Y^{i} e_{l-i}
$$

which is DE PRIL's (1986) recursion for the moments of the compound distribution when $M \sim R_{1}\left(a \frac{\rho_{2}}{1-a \rho_{1}}, b \frac{\rho_{2}}{1-a \rho_{1}}\right)$.

\section{BIVARIATE $R_{2}$ DISTRIBUTIONS}

In this section we consider the following situations:

B. $N=R_{0}+R_{1}$ and $M=R_{0}+R_{2}$, where $R_{0}, R_{1}$ and $R_{2}$ are mutually independent and $R_{j} \sim R_{1}\left(a_{j}, b_{j}\right)$.

\subsection{Bivariate distributions}

The case where $R_{0}, R_{1}$ and $R_{2}$ are independently Poisson distributed has previously been considered in the literature. TEICHER (1954) (see also JoHNSON and KoTz, 1969 , p. 298) showed that this bivariate Poisson distribution satisfies the recurrence relations

$$
\begin{aligned}
& n p(n, m)=\lambda_{1} p(n-1, m)+\lambda_{0} p(n-1, m-1), \\
& m p(n, m)=\lambda_{2} p(n, m-1)+\lambda_{0} p(n-1, m-1),
\end{aligned}
$$

where $\lambda_{0}, \lambda_{1}$ and $\lambda_{2}$ denote the Poisson parameters for $R_{0}, R_{1}$ and $R_{2}$, respectively. Holgate (1954) treated the estimation problems for this distribution.

Since the bivariate Poisson distribution appears as a special case of condition B with $a_{j}=0$ and $b_{j}=\lambda_{j}$, the following result is seen to generalize that of TEICHER (1954). 
Theorem 3.1 Under condition $B$ it holds that

$$
\begin{aligned}
p(n, m)= & \left(a_{0}+\frac{b_{0}}{n}\right) p(n-1, m-1)+\left(a_{1}+\frac{b_{1}}{n}\right) p(n-1, m) \\
& -\left(a_{0} a_{1}+\frac{a_{1} b_{0}+b_{1} a_{0}}{n}\right) p(n-2, m-1), n \geq 1, \\
p(n, m)= & \left(a_{0}+\frac{b_{0}}{m}\right) p(n-1, m-1)+\left(a_{2}+\frac{b_{2}}{m}\right) p(n, m-1) \\
& -\left(a_{0} a_{2}+\frac{a_{2} b_{0}+b_{2} a_{0}}{m}\right) p(n-1, m-2), m \geq 1,
\end{aligned}
$$

where $p(0,0)=\pi_{0} \pi_{1} \pi_{2}, \pi_{j}=\mathrm{P}\left(R_{j}=0\right)$, and $p(n, m)=0$ when $n<0$ or $m<0$.

Proof. The pgf for $(N, M)$ is in this case

$$
\varphi(s, t)=\mathrm{E}\left[s^{N} t^{M}\right]=\mathrm{E}\left[(s t)^{R_{0}} s^{R_{1}} t^{R_{2}}\right]=\psi_{0}(s t) \psi_{1}(s) \psi_{2}(t)
$$

where $\psi_{j}$ denotes the pgf for $R_{j} \sim R_{1}\left(a_{j}, b_{j}\right)$. Differentiating (3.3) with respect to $s$ and making use of (2.3) yields

$$
\begin{aligned}
(1 & \left.-a_{1} s-a_{0} s t+a_{0} a_{1} t s^{2}\right) \frac{d}{d s} \varphi(s, t) \\
& =\left[t\left(1-a_{1} s\right)\left(a_{0}+b_{0}\right)+\left(a_{1}+b_{1}\right)\left(1-a_{0} s t\right)\right] \varphi(s, t),
\end{aligned}
$$

and with $\varphi(s, t)=\sum_{n, m \geq 0} p(n, m) s^{n} t^{m}$ we compare the coefficients of $s^{n-1} t^{m}$ in this equation for $n \geq 1$ to obtain

$$
\begin{aligned}
n p(n, m)-a_{1}(n-1) p(n-1, m)-a_{0}(n-1) p(n-1, m-1) \\
\quad+a_{0} a_{1}(n-2) p(n-2, m-1) \\
=\left(a_{1}+b_{1}\right) p(n-1, m)+\left(a_{0}+b_{0}\right) p(n-1, m-1) \\
\quad-\left[a_{1}\left(a_{0}+b_{0}\right)+a_{0}\left(a_{1}+b_{1}\right)\right] p(n-2, m-1) .
\end{aligned}
$$

This verifies (3.1), and (3.2) follows analogously.

QED

Remark 1. Note from the proof of Theorem 3.1 that (3.1) only requires that $R_{0}$ and $R_{1}$ are of class $R_{1}$. If $p(0, m)$ is known for $m=0,1, \ldots$, the bivariate $\mathrm{pf} p(n, m)$ may therefore be calculated recursively from (3.1) even when $R_{2}$ does not belong to $R_{1}$. A similar remarks holds if $R_{1}$ does not belong to $R_{1}$.

From the recurrence relation in Theorem 3.1 we easily obtain the following recursion for the corresponding bivariate compound distribution: 
Theorem 3.2 Assume that condition B holds true. Then

$$
g(0,0)=\psi_{0}\left(f_{1}(0) f_{2}(0)\right) \psi_{1}\left(f_{1}(0)\right) \psi_{2}\left(f_{2}(0)\right),
$$

where $\psi_{j}(z)$ is the pgf for $R_{j}$. For $x \leq 1$,

$$
\begin{aligned}
g(x, y)= & \sum_{u=0}^{x}\left(a_{1}+\frac{b_{1} u}{x}\right) f_{1}(u) g(x-u, y) \\
& +\sum_{u=0}^{x} \sum_{v=0}^{y}\left(a_{0}+\frac{b_{0} u}{x}\right) f_{1}(u) f_{2}(v) g(x-u, y-v) \\
& -\sum_{u=0}^{x} \sum_{v=0}^{y}\left(a_{9} a_{1}+\frac{\left(a_{0} b_{1}+b_{0} a_{1}\right) u}{2 x}\right) f_{1}^{* 2}(u) f_{2}(v) g(x-u, y-v)
\end{aligned}
$$

and for $y \geq 1$,

$$
\begin{aligned}
g(x, y)= & \sum_{\nu=0}^{y}\left(a_{2}+\frac{b_{2} v}{y}\right) f_{2}(v) g(x, y-v) \\
& +\sum_{\nu=0}^{y} \sum_{u=0}^{x}\left(a_{0}+\frac{b_{0} v}{y}\right) f_{2}(v) f_{1}(u) g(x-u, y-v) \\
& -\sum_{\nu=0}^{y} \sum_{u=0}^{x}\left(a_{0} a_{2}+\frac{\left(a_{0} b_{2}+b_{0} a_{2}\right) v}{2 y}\right) f_{2}^{* 2}(v) f_{1}(u) g(x-u, y-v) .
\end{aligned}
$$

Proof. We have that

$$
g(0,0)=\sum_{n, m=0}^{\infty} p(n, m) f_{1}(0)^{n} f_{2}(0)^{m}=\varphi\left(f_{1}(0), f_{2}(0)\right)
$$

where $\varphi(s, t)$ is the pgf for $(N, M)$, and (3.4) therefore follows from (3.3).

Using (1.5) we have for $i \geq 1$ and $j \geq 0$ that

$$
\sum_{n=i, m=j}^{\infty} \frac{1}{n} p(n-i, m-j) f_{1}^{* n}(x) f_{2}^{*_{m}}(y)=\sum_{u=0}^{x} \sum_{\nu=0}^{y} \frac{u}{i x} f_{1}^{*_{i}}(u) f_{2}^{* j}(v) g(x-u, y-v) .
$$

Multiplying (3.1) with $f_{1}^{*_{n}}(x) f_{2}^{* m}(y)$ and summing over $n, m$ then yields (3.5), and (3.6) follows analogously from (3.2).

Remark 2. Since (3.5) was obtained by use of (3.1), it follows from Remark 1 that $g(x, y)$ may be calculated recursively from $(3.5)$ when $g(0, y)$ is known for $y=0,1$, ..., even if $R_{2}$ does not belong to $R_{1}$. A similar remark holds if $R_{1}$ does not belong to $R_{1}$.

It is seen from (3.5) and (3.6) that the number of multiplications involved with the calculation of $g(u, v)$ for $(u, v) \leq(x, y)$ is of order $O\left(x^{2} y^{2}\right)$. If the severity 
distributions $f_{1}$ and $f_{2}$ have bounded support, as will typically be the case in practical applications, the number of multiplications will be of order $O(x y)$.

For the bivariate Poisson distribution corresponding to $a_{j}=0$ and $b_{j}=\lambda_{j}$, the recursive formulas in Theorem 3.2 simplify substantially. In this case we have,

$$
\begin{aligned}
g(x, y)= & \frac{\lambda_{1}}{x} \sum_{u=1}^{x} u f_{1}(u) g(x-u, y) \\
& +\frac{\lambda_{0}}{x} \sum_{u=1}^{x} \sum_{v=0}^{y} u f_{1}(u) f_{2}(v) g(x-u, y-v), x \geq 1, \\
g(x, y)= & \frac{\lambda_{2}}{y} \sum_{v=1}^{y} v f_{2}(v) g(x, y-v) \\
& +\frac{\lambda_{0}}{y} \sum_{u=0}^{x} \sum_{v=1}^{y} v f_{1}(u) f_{2}(v) g(x-u, y-v), y \geq 1 .
\end{aligned}
$$

\subsection{Marginal and conditional distributions}

SUNDT (1992) considered the class $R_{k}$ of counting distributions satisfying

$$
q(n)=\sum_{i=1}^{k}\left(\alpha_{i}+\frac{\beta_{i}}{n}\right) q(n-i),
$$

for suitable constants $\alpha_{i}$ and $\beta_{i}$, and showed that if $q_{1} \in R_{k}, q_{2} \in R_{l}$, then the convolution $q_{1} * q_{2}$ belongs to $R_{k+l}$. From this we observe that the marginal distributions of $N$ and $M$ belong to $R_{2}$ under condition $\mathrm{B}$, and Theorem 3.2 may therefore be viewed as a bivariate extension of SUNDT (1992, Theorem 9). The representation (3.7) for the marginal distribution of $N$ can be found in SUNDT (1992, Corollary 4), or may be obtained by summing (3.1) over $m$. Similarly, a recursion for the marginal distribution of $X$ can be obtained from SundT (1992, Theorem 9), or by summing (3.5) over $y$.

The following result gives a recursion for the auxiliary quantities $\tilde{\mu}_{l, x}$. Since the marginal pf $g(x)$ can be calculated recursively from SUNDT (1992, Theorem 9) we then have a recursive procedure for calculating the conditional moments $\mu_{l, x}=$ $\tilde{\mu}_{l, x} / g(x)$. Theorem 3.3 follow by straightforward calculations, and the details can be found in Appendix A.

Theorem 3.3 Assume that condition B holds true. With

$$
\begin{aligned}
& c_{j}(l, i)=a_{j}\left(\begin{array}{l}
l \\
i
\end{array}\right)+b_{j}\left(\begin{array}{c}
l-I \\
i
\end{array}\right), j=0,2, \\
& d(l, i)=a_{0} c_{2}(l, i)+a_{2} c_{0}(l, i),
\end{aligned}
$$


it holds for $l \geq 1$ that

$$
\begin{aligned}
& \left(1-a_{2}\right)\left(1-a_{0} f_{1}(0)\right) \tilde{\mu}_{l, x}=\sum_{i=0}^{l-1} c_{2}(l, i) \tilde{\mu}_{i, x} e_{l-i}+a_{0}\left(1-a_{2}\right) \sum_{u=1}^{x} f_{1}(u) \tilde{\mu}_{l, x-u} \\
& \quad+\sum_{u=0}^{x} f_{1}(u) \sum_{i=0}^{l-1} \tilde{\mu}_{i, x-u}\left[c_{0}(l, i) e_{l-i}-\frac{1}{2} d(l, i) e_{l-i}^{* 2}\right], \\
& e_{j}=\sum_{v=0}^{\infty} v^{j} f_{2}(v) \text { and } e_{j}^{* 2}=\sum_{\nu=0}^{\infty} v^{j} f_{2}^{* 2}(v) .
\end{aligned}
$$

Remark 3. The recursion in Theorem 3.3 is obtained from (3.6), and is according to Remarks 1 and 2 therefore also valid when $R_{1}$ does not belong to $R_{1}$.

\section{4. bivariate mixed Poisson distributions}

Consider the following situation:

C. $N$ and $M$ are conditionally independent given $\theta=\vartheta$, with pf

$$
p_{\vartheta}(n, m)=\frac{\left(\vartheta \lambda_{1}\right)^{n}}{n !} e^{-\vartheta \lambda_{1}} \frac{\left(\vartheta \lambda_{2}\right)^{m}}{m !} e^{-\vartheta \lambda_{2}}
$$

The parameter $\theta \in\left[\sigma_{1}, \sigma_{2}\right], 0 \leq \sigma_{1}<\sigma_{2} \leq \infty$, has a density $u$ which satisfies

$$
\frac{d}{d \vartheta} \log u(\vartheta)=\frac{\sum_{i=0}^{k} a_{i} \vartheta^{i}}{\sum_{i=0}^{k} b_{i} \vartheta^{i}}
$$

for suitable constants $a_{i}$ and $b_{i}$, and

$$
\sum_{i=0}^{k} b_{i} \vartheta^{i} u(\vartheta) \rightarrow 0, \vartheta \rightarrow \sigma_{1}, \sigma_{2}
$$

Let

$$
g_{\vartheta}^{(j)}(x)=\sum_{n=0}^{\infty} \frac{\left(\vartheta \lambda_{j}\right)^{n}}{n !} e^{-\vartheta \lambda_{j}} f_{j}^{* n}(x), j=1,2,
$$

denote the conditional pf's of $X$ and $Y$ and let

$$
g_{\vartheta}(x, y)=\sum_{n . m=0}^{x} p_{\vartheta}(n, m) f_{1}^{* n}(x) f_{2}^{*_{m}}(y)=g_{\vartheta}^{(1)}(x) g_{\vartheta}^{(2)}(y),
$$

denote the joint conditional pf of $(X, Y)$. Finally, introduce the auxiliary functions

$$
h_{i}(x, y)=\int_{\sigma_{1}}^{\sigma_{2}} \vartheta^{i} g_{\vartheta}(x, y) u(\vartheta) d \vartheta
$$

and note that $g(x, y)=h_{0}(x, y)$. 


\subsection{Bivariate distributions}

The following recursion for the function $h_{i}(x, y)$, and hence the pf $g(x, y)$, is a bivariate extension of the recursion presented in HESSELAGER (1993).

Theorem 4.1 Under condition $C$ it holds that

$$
h_{i}(0,0)=\int_{\sigma_{1}}^{\sigma_{2}} \vartheta^{i} e^{-\lambda \cdot(1-\tilde{f}(0)) \vartheta} u(\vartheta) d \vartheta
$$

with $\lambda .=\lambda_{1}+\lambda_{2}$, and $\tilde{f}(0)=\frac{\lambda_{1} f_{1}(0)+\lambda_{2} f_{2}(0)}{\lambda_{1}+\lambda_{2}}$. For $i=0, \ldots, \mathrm{k}-1$,

$$
\begin{aligned}
& h_{i}(x, y)=\lambda_{1} \sum_{u=1}^{x} \frac{u}{x} f_{1}(u) h_{i+1}(x-u, y), x \geq 1, \\
& h_{i}(x, y)=\lambda_{2} \sum_{v=1}^{y} \frac{v}{y} f_{2}(v) h_{i+1}(x, y-v), y \geq 1,
\end{aligned}
$$

and

$$
\begin{aligned}
c_{k} h_{k}(x, y)= & \lambda_{1} \sum_{u=1}^{x} f_{1}(u) \sum_{i=0}^{k} b_{i} h_{i}(x-u, y)+\lambda_{2} \sum_{v=1}^{y} f_{2}(y) \sum_{i=0}^{k} b_{i} h_{i}(x, y-v) \\
& +\sum_{i=0}^{k-1} h_{i}(x, y)\left[(i+1) b_{i+1}-c_{i}\right],
\end{aligned}
$$

where $c_{i}=\lambda \cdot(1-\tilde{f}(0)) b_{i}-a_{i}$.

With initial values $h_{i}(0,0)$ one calculates $h_{0}(x, 0), \ldots, h_{k-1}(x, 0)$ from $(4.7)$ and $h_{k}(x, 0)$ from $(4.9)$ for $x=1,2, \ldots$ For $y=1,2, \ldots$ one calculates $h_{0}(0, y), \ldots$, $h_{k-1}(0, y)$ from $(4.8)$ and $h_{k}(0, y)$ from $(4.9)$. For $(x, y) \geq(1,1)$ one may then use either (4.7) or (4.8) together with (4.9). The recursion is seen to be of order $O(x y(x+y))$, reducing to $O(x y)$, when the supports of $f_{1}$ and $f_{2}$ are finite.

Proof of Theorem 4.1. Since $g_{\vartheta}^{(j)}$ is compound Poisson with parameter $\lambda_{j} \vartheta$ it holds that $g_{\vartheta}^{(j)}(0)=e^{-\vartheta \lambda_{j}\left(1-f_{j}(0)\right)}$, and $g_{\vartheta}(0,0)=g_{\vartheta}^{(1)}(0) g_{\vartheta}^{(2)}(0)=e^{-\vartheta \lambda .(1-\tilde{f}(0))}$ with $\lambda$. and $\tilde{f}(0)$ as stated, and (4.6) then follows from the definition (4.5).

Under the Poisson assumption of condition $\mathrm{C}$, the conditional pf $g_{i}^{(j)}(x)$ satisfies the Panjer recursion,

$$
g_{\vartheta}^{(j)}(z)=\vartheta \lambda_{j} \sum_{u=1}^{z} \frac{u}{z} f_{j}(u) g_{\vartheta}^{(j)}(z-u), z \geq 1, j=1,2,
$$


and from (4.4) we than have that

$$
\begin{aligned}
g_{\vartheta}(x, y) & =g_{\vartheta}^{(2)}(y) \vartheta \lambda_{1} \sum_{u=1}^{x} \frac{u}{x} f_{1}(u) g_{\vartheta}^{(1)}(x-u) \\
& =\vartheta \lambda_{1} \sum_{u=1}^{x} \frac{u}{x} f_{1}(u) g_{\vartheta}(x-u, y), x \geq 1,
\end{aligned}
$$

and similarly,

$$
g_{\vartheta}(x, y)=\vartheta \lambda_{2} \sum_{\nu=1}^{y} \frac{v}{y} f_{2}(v) g_{\vartheta}(x, y-v), y \geq 1 .
$$

By multiplying this expression with $\vartheta^{i} u(\vartheta)$, and integrating over $\vartheta$ we obtain (4.7) and (4.8).

Differentiating (4.1) wrt $\vartheta$ yields

(4.10) $\frac{d}{d \vartheta} p_{\vartheta}(n, m)=\lambda_{1} p_{\vartheta}(n-1, m)+\lambda_{2} p_{\vartheta}(n, m-1)-\lambda . p_{\vartheta}(n, m)$.

According to (4.2) it holds that $u(\vartheta) \sum_{i=0}^{k} a_{i} \vartheta^{i}=u^{\prime}(\vartheta) \sum_{i=0}^{k} b_{i} \vartheta^{i}$, and partial integration using (4.10) yields

$$
\begin{aligned}
& \int_{\sigma_{1}}^{\sigma_{2}} \sum_{i=0}^{k} a_{i} \vartheta^{i} p_{\vartheta}(n, m) u(\vartheta) d \vartheta=\int_{\sigma_{1}}^{\sigma_{2}} \sum_{i=0}^{k} b_{i} \vartheta^{i} p_{\vartheta}(n, m) u^{\prime}(\vartheta) d \vartheta \\
&=\left[\sum_{i=0}^{k} b_{i} \vartheta^{i} p_{\vartheta}(n, m) u(\vartheta)\right]_{\sigma_{1}}^{\sigma_{2}}-\int_{\sigma_{1}}^{\sigma_{2}} \sum_{i=1}^{k} i \vartheta^{i-1} b_{i} u(\vartheta) p_{\vartheta}(n, m) d \vartheta \\
& \quad-\int_{\sigma_{1}}^{\sigma_{2}} \sum_{i=0}^{k} b_{i} \vartheta^{i} u(\vartheta)\left[\lambda_{1} p_{\vartheta}(n-1, m)+\lambda_{2} p_{\vartheta}(n, m-1)-\lambda \cdot p_{\vartheta}(n, m)\right] d \vartheta \\
&=\int_{\sigma_{1}}^{\sigma_{2}} \sum_{i=0}^{k-1}(i+1) \vartheta^{i} b_{i+1} u(\vartheta) p_{\vartheta}(n, m) d \vartheta \\
&-\int_{\sigma_{1}}^{\sigma_{2}} \sum_{i=0}^{k} b_{i} \vartheta^{i} u(\vartheta)\left[\lambda_{1} p_{\vartheta}(n-1, m)+\lambda_{2} p_{\vartheta}(n, m-1)-\lambda \cdot p_{\vartheta}(n, m)\right] d \vartheta,
\end{aligned}
$$

where the last equality follows from (4.3). Multiply on both sides with $f_{1}^{* n}(x) f_{2}^{* m}(y)$ and sum over $(n, m)$ to obtain

$$
\text { (4.11) } \begin{aligned}
\sum_{i=0}^{k} a_{i} h_{i}(x, y)= & -\sum_{i=0}^{k-1}(i+1) b_{i+1} h_{i}(x, y)-\lambda_{1} \sum_{u=0}^{x} f_{1}(u) \sum_{i=0}^{k} b_{i} h_{i}(x-u, y) \\
& -\lambda_{2} \sum_{\nu=0}^{y} f_{2}(\nu) \sum_{i=0}^{k} b_{i} h_{i}(x, y-\nu)+\lambda . \sum_{i=0}^{k} b_{i} h_{i}(x, y) .
\end{aligned}
$$

By rearranging terms we then arrive at (4.9). 
The expression (4.6) for the initial values is analogous to the expression given by HESSELAGER (1993) for the univariate case, and the reader will in that paper find explicit formulas for (4.6) for a number of cases.

It should be noted that the gamma mixing density satisfies (4.2), and the corresponding mixed Poisson distribution can be shown to be the bivariate negative binomial distribution treated in Section 2. For this case, the recursion in Theorem 2.2 is simpler than the one given in Theorem 4.1, although they are both of the same order.

The condition (4.3) may be dropped without serious consequences, and the identity (4.9) will in this case contain an additional term as in Theorem 1 of Hesselager (1993). In all of the examples considered in Hesselager (1993), (4.3) is however fulfilled.

Univariate mixed Poisson distributions with a mixing density which satisfies (4.2) were studied by WILLMOT (1993) who obtained a recurrence relation for the counting distribution and also investigated a number of special cases where (4.2) holds true. A recursion for the bivariate counting distribution is obtaining by letting $f_{1}(1)=f_{2}(1)=1$ in Theorem 4.1. In this case we may eliminate the auxiliary functions $h_{i}(n, m)$ for $i \geq 1$ and obtain a recurrence relation for the pf $p(n, m)=$ $h_{0}(n, m)$, analogous to that of WiLLMot (1993):

Theorem 4.2 Under condition $C$ it holds for $n>k$ that

(4.12) $\sum_{i=0}^{k+1} \lambda_{1}^{i}(n-i) ! p(n-i, m)\left\{a_{k-i}-\lambda . b_{k-i}+(n+m+1-i) b_{k+1-i}\right\}=0$.

and for $m>k$,

(4.13) $\sum_{i=0}^{k+1} \lambda_{2}^{i}(m-i) ! p(n, m-i)\left\{a_{k-i}-\lambda . b_{k-i}+(n+m+1-i) b_{k+1-i}\right\}=0$,

with the convention that $a_{-1}=b_{-1}=b_{k+1}=0$.

Proof. When $f_{1}(1)=f_{2}(1)=1$, the relations (4.7), (4.8) become

$$
h_{i}(n, m)=\frac{\lambda_{1}}{n} h_{i+1}(n-1, m), \quad h_{i}(n, m)=\frac{\lambda_{2}}{m} h_{i+1}(n, m-1),
$$

Repeated use of the first relation gives $h_{i}(n, m)=\lambda_{1}^{-i}(n+i)^{(i)} p(n+i)$, where $(n+i)^{(i)}=(n+i) ! / n !$. By inserting into (4.11), which in this case becomes

$$
\text { (4.15) } \begin{aligned}
\sum_{i=0}^{k} a_{i} h_{i}(n, m)= & -\sum_{i=0}^{k-1}(i+1) b_{i+1} h_{i}(n, m)-\lambda_{1} \sum_{i=0}^{k} b_{i} h_{i}(n-1, m) \\
& -\lambda_{2} \sum_{i=0}^{k} b_{i} h_{i}(n, m-1)+\lambda . \sum_{i=0}^{k} b_{i} h_{i}(n, m),
\end{aligned}
$$


we then obtain after a little rearrangement that

$$
\begin{aligned}
& \sum_{i=0}^{k}\left(a_{k-i}-\lambda \cdot b_{k-i}\right) \lambda_{1}^{i}(n-i) ! p(n-i, m)= \\
& \quad-\sum_{i=1}^{k}(k-i+1) b_{k-i+1} \lambda_{1}^{i}(n-i) ! p(n-i, m) \\
& \quad-\lambda_{1} \sum_{i=0}^{k} b_{k-i} \lambda_{1}^{i}(n-k)(n-i-1) ! p(n-i-1, m) \\
& \quad-\lambda_{2} \sum_{i=0}^{k} b_{k-i} \lambda_{1}^{i}(n-i) ! p(n-i, m-1) .
\end{aligned}
$$

From (4.14) it follows that

$$
p(n-i, m-1)=\frac{\lambda_{1}}{n-i} h_{1}(n-i-1, m-1)=\frac{\lambda_{1} m}{(n-i) \lambda_{2}} p(n-i-1, m),
$$

which inserted into the last term in (4.16) leads to (4.12) after rearranging terms. The relation (4.13) follows analogously.

QED

The reader will notice from the proof of Theorem 4.2 that there is a whole variety of recurrence relations for the bivariate pf $p(n, m)$. From (4.14) we observe that

$$
h_{i}(n, m)=\frac{(n+j)^{(j)}}{\lambda_{1}^{j}} \frac{(m+i-j)^{(i-j)}}{\lambda_{2}^{i-j}} p(n+j, m+i-j)
$$

for arbitrary $j=0, \ldots, i$, which together with (4.15) also will give a recurrence relation. In particular (4.16) is such a relation, which in fact is the bivariate extension of WiLLMOT's (1993) recurrence relation for the univariate case, as one may verify by summing (4.16) over $m \geq 0$.

\subsection{Marginal and conditional distributions}

The marginal distributions are in this case the mixed Poisson and the corresponding compound mixed Poisson distributions considered by WILLMOT (1993) and HessELAGER (1993).

Since $N$ and $M$ are conditionally independent given $\theta=\vartheta$, it follows that

$$
p(m \mid n)=\int \frac{\left(\lambda_{2} \vartheta\right)^{m}}{m !} e^{-\lambda_{2} \vartheta} u(\vartheta \mid n) d \vartheta
$$

where $u(\vartheta \mid n)$ is the posterior density for $\theta$ given $N=n$. When the prior density $u(\vartheta)$ satisfies (4.2), it was verified in HESSELAGER (1993) that $u(\vartheta \mid n)$ also satisfies 
(4.2) with updated constants $\tilde{a}_{i}, \tilde{b}_{i}$. Thus, the conditional compound distribution of $Y$ given $N=n$ may be calculated recursively by use of the recursion from Hesselager (1993).

For the conditional distribution of $Y$ given $X=x$ we may multiply (4.8) and (4.9) by $y^{l}$ and sum over $y$ to obtain a recursive algorithm for the auxiliary function $\tilde{\mu}_{l, x}^{(i)}$ $=\sum_{y} y^{l} h_{i}(x, y)$. This, in particular, will give a recursion for the function $\tilde{\mu}_{l, x}=\tilde{\mu}_{l, x}^{(0)}$, and hence for the conditional moments $\mu_{l, x}=\tilde{\mu}_{l, x} / g(x)$. We state the result without proof :

Theorem 4.3 With $c_{i}=\lambda_{I} b_{i}\left(1-f_{1}(0)\right)-a_{i}$ and $e_{j}=\sum_{v} v^{j} f_{2}(v)$ it holds that

$$
\begin{gathered}
\tilde{\mu}_{l, x}^{(i)}=\lambda_{2} \sum_{j=0}^{l-1}\left(\begin{array}{c}
l-1 \\
j
\end{array}\right) e_{l-j} \tilde{\mu}_{j, x}^{(i+1)}, i=0, \ldots, k-1, \\
c_{k} \tilde{\mu}_{l, x}^{(k)}=\lambda_{2} \sum_{i=0}^{k} \sum_{j=0}^{k-1}\left(\begin{array}{l}
l \\
j
\end{array}\right) \tilde{\mu}_{j, x}^{(i)} e_{l-j}+\sum_{i=0}^{k-1}\left(b_{i+1}(i+1)-c_{i}\right) \tilde{\mu}_{l, x}^{(i)} .
\end{gathered}
$$

APPENDIX A

Proof of Theorem 2.4. For $l \geq 1$, multiply (2.6) by $y^{l}$ and sum over $y$ to obtain

$$
\begin{aligned}
\tilde{\mu}_{l, x}= & \sum_{y=1}^{\infty} y^{l} g(x, y) \\
= & \rho_{2} \sum_{y=1}^{\infty} \sum_{\nu=0}^{y}\left(a y^{l}+b v y^{l-1}\right) f_{2}(v) g(x, y-v) \\
& +a \rho_{1} \sum_{y=1}^{\infty} \sum_{u=0}^{x} f_{1}(u) y^{l} g(x-u, y) \\
= & \rho_{2} \sum_{v=0}^{\infty} \sum_{y=0}^{\infty}\left(a(y+v)^{l}+b v(y+v)^{l-1}\right) f_{2}(v) g(x, y) \\
& +a \rho_{1} \sum_{u=0}^{x} f_{1}(u) \tilde{\mu}_{l, x-u} \\
= & \rho_{2} a \sum_{i=0}^{l}\left(\begin{array}{l}
l \\
i
\end{array}\right) \tilde{\mu}_{i, x} e_{l-i}+\rho_{2} b \sum_{i=0}^{l-1}\left(\begin{array}{c}
l-1 \\
i
\end{array}\right) \tilde{\mu}_{i, x} e_{l-i}+a \rho_{1} \sum_{u=0}^{x} f_{1}(u) \tilde{\mu}_{l, x-u} .
\end{aligned}
$$


Proof of Theorem 3.3. Multiply (3.6) by $y^{l}$ and sum over $y$ to obtain for $l \geq 1$ that

$$
\begin{aligned}
& \tilde{\mu}_{l, x}=\sum_{y=1}^{\infty} y^{l} g(x, y) \\
& =\sum_{v, y=0}^{\infty}\left[a_{2}(y+v)^{l}+b_{2} v(v+y)^{l-1}\right] f_{2}(v) g(x, y) \\
& +\sum_{u=0}^{x} \sum_{\nu, y=0}^{x}\left[a_{0}(y+v)^{l}+b_{0} v(v+y)^{l-1}\right] f_{1}(u) f_{2}(v) g(x-u, y) \\
& -a_{0} a_{2} \sum_{u=0}^{x} \sum_{v, y=0}^{x}(y+v)^{\prime} f_{2}^{* 2}(v) f_{1}(u) g(x-u, y) \\
& -\frac{b_{0} a_{2}+a_{0} b_{2}}{2} \sum_{u=0}^{x} \sum_{\nu, y=0}^{\infty} v(v+y)^{l-1} f_{2}^{* 2}(v) f_{1}(u) g(x-u, y) \\
& =a_{2} \sum_{i=0}^{l}\left(\begin{array}{l}
l \\
i
\end{array}\right) \tilde{\mu}_{i, x} e_{l-i}+b_{2} \sum_{i=0}^{l-1}\left(\begin{array}{c}
l-1 \\
i
\end{array}\right) \tilde{\mu}_{i, x} e_{l-i} \\
& +\sum_{u=0}^{x} f_{1}(u)\left[a_{0} \sum_{i=0}^{l}\left(\begin{array}{l}
l \\
i
\end{array}\right) \tilde{\mu}_{i, x-u} e_{l-i}+b_{0} \sum_{i=0}^{l-1}\left(\begin{array}{c}
l-1 \\
i
\end{array}\right) \tilde{\mu}_{i, x-u} e_{l-i}\right] \\
& -a_{0} a_{2} \sum_{u=0}^{x} f_{1}(u) \sum_{i=0}^{l}\left(\begin{array}{l}
l \\
i
\end{array}\right) \tilde{\mu}_{i, x-u} e_{l-i}^{* 2} \\
& -\frac{\left(b_{0} a_{2}+a_{0} b_{2}\right)}{2} \sum_{u=0}^{x} f_{1}(u) \sum_{i=0}^{l-1}\left(\begin{array}{c}
l-1 \\
i
\end{array}\right) \tilde{\mu}_{i, x-u} e_{l-i}^{* 2} \\
& =a_{2} \tilde{\mu}_{l, x}+\sum_{i=0}^{l-1} c_{2}(l, i) \tilde{\mu}_{i, x} e_{l-i}+a_{0}\left(l-a_{2}\right) \sum_{u=0}^{x} f_{l}(u) \tilde{\mu}_{l, x-u} \\
& +\sum_{u=0}^{x} f_{1}(u) \sum_{i=0}^{l-1} \tilde{\mu}_{i, x-u}\left[c_{0}(l, i) e_{l-i}-\frac{1}{2} d(l, i) e_{l-i}^{* 2}\right],
\end{aligned}
$$

and the result follows upon rearranging terms.

\section{REFERENCES}

DE PRIL, N. (1986) Moments of a class of compound distributions. Scand. Actuarial J., 1986, 117-120.

GUlDBERG, A. (1934) On discontinuous frequency functions of two variables. Skandinavisk Aktuarietidskrift, 1934, 89-117.

Hessel.Ager, O. (1993) A recursive procedure for calculation of some mixed compound Poisson distributions. Working paper, 115, Laboratory of Actuarial Math., Copenhagen. (To appear in Scand. Actuarial J.). 
HESSELAGER, O. (1994) A recursive procedure for calculation of some compound distributions. ASTIN Bulletin, 24, 19-32.

Holgate, P. (1954) Estimation for the bivariate Poisson distribution. Biometrika, 51, 241-245.

Johnson, N.L. and Kotz, S. (1969) Distributions in Statistics: Discrete distributions. Wiley, New York.

Kocherlakota, S. and Kocherlakota, K. (1992) Bivariate Discrete Distributions, Marcel Dekker, New York.

PANJER, H.H. (1981) Recursive evaluation of a family of compound distributions. ASTIN Bulletin, 12, 22-26.

Panjer, H.H. and Willmot, G. (1982) Recursions for compound distributions. ASTIN Bulletin. 13, $1-11$.

Panjer, H.H. and Willmot, G. (1992) Insurance Risk Models. Society of Actuaries, Schaumburg.

Papageorgiou, H. (1984) On recursive formulas for aggregate claims. Scand. Actuarial J., 1984. 102-104.

SCHRÖTER, K.J. (1990) On a class of counting distributions and recursions for related compound distributions. Scand. Actuarial J., 1990, 161-175.

SundT, B. (1992) On some extensions of Panjer's class of counting distributions. ASTIN Bulletin, 22, 61-80.

TeICHER, H. (1954) On the multivariate Poisson distribution. Skandinavisk Aktuarietidskrift, 1954, 1-9.

WANG, S. and SoBrero, M. (1994) Further results on Hesselager's recursive procedure for calculation of some compound distributions. ASTIN Bulletin, 24, 160-166.

Willmot, G. (1993) On recursive evaluation of mixed Poisson probabilities and related quantities. Scand. Actuarial J., 1993, 114-133.

Ole HesSElAGER

Laboratory of Actuarial Mathematics

University of Copenhagen

Universitetsparken 5

DK-2100 Copenhagen $\emptyset$ 